\title{
CONSTRAINT OPTIMAL POWER FLOW BASED ON ANT COLONY OPTIMIZATION
}

Ali Qasim ${ }^{1}$

\section{${ }^{*}$ Dr. Layth Tawfeeq Al-Bahrani ${ }^{2}$}

1) M.Sc. Student, Electrical Engineering Department, Collage of Engineering, Muatansiriayh University, Baghdad, Iraq

2) Electrical Engineering Department, Collage of Engineering, Muatansiriayh University, Baghdad, Iraq

\begin{abstract}
The Ant Colony Optimization ACO technique simulates the real ant colonies movements to find the best solution for huge optimization problems according to a group of agents called ants. In this article, the Ant Colony Optimization is used to solve a single objective function of Optimal Power Flow OPF problem with satisfied a set of the system constraints. ACO technique minimizes the objective function based on choosing an optimal control variables from their limits satisfying the state variables constraints. The performance of ACO in solving OPF problems has been demonstrated on the IEEE-30 bus system with different objective functions such as fuel cost minimization, voltage profile improvement and active power losses minimization. The results acquired through the simulation were good when compared with other optimization techniques reported in the literature. The outcomes suggest that this technique is effectively resolving the power system with vastly ranged network.
\end{abstract}

Keywords: Constraint Optimal Power Flow, Ant Colony Optimization

\section{Introduction}

The Optimal Power Flow OPF is one of the prevailing problems in power system [1]. Since the 1960s, the optimal power flow has been expressing a constrained economic power dispatch problem [2], it has been tested and studied for the purpose of obtaining an optimal solution for minimizing an objective function through regulating power system control variables while still meeting the operational and physical constraints [3]. The control variables such as the generator real power, the generator bus magnitude voltage, the reactive power of shunt compensator and the tap ratios of transformer are selected from their limits of minimum and maximum values. The problem of the OPF can be solved into two techniques, the first optimization technique is well-known as conventional or classical technique such as linear programming, nonlinear programming, quadratic programming, newton method and interior point method,... etc. [4, 5]. These classical techniques provide a good result, but it suffer from many problem such as the high dimensionality, nonlinearity, non-differentiability and the noncontinuously that exist in the OPF. This type of optimization technique transition the optimal power flow into a complex problem and may be not able to present an optimal solution. In order to overcome some complications of the conventional optimization techniques, the second type of optimization techniques which are

*Corresponding Author: Laith1973a@gmail.com 
renowned as the artificial intelligence optimization techniques has been used such as Genetic Algorithm GA, Artificial Neural Network ANN, Differential Evolution DE, Particle Swarm Optimization PSO, Artificial Bee Colony $\mathrm{ABC}$ and Ant Colony Optimization $\mathrm{ACO}, \ldots$ etc. These optimization methods are inspired from the collective behaviors of animals and evolutionary algorithms [1]. The propose algorithm ACO is suggested to solve the constraint optimal power flow problem using the IEEE 30-bus test system to minimized the objective functions of OPF.

\section{Optimal Power Flow (OPF) Problem Formulation}

The OPF is the process of maximizing optimization with a number of objectives. The nature of the solution depends upon the accuracy of the studied mathematical model. Objectives must be tested for its practicality with potential solutions. Generally, OPF problem can be represented according to the following form:

minimum $F(x, \mu)$

$g(x, \mu)=0$

$h(x, \mu) \leq 0$

$F(x, \mu)$ is the objective function; $g(x, \mu)$ is a function that represents the equality constraints; $h(x, \mu)$ is a function that represents the inequality constraints; $x$ is the vector of dependent state variables; $\mu$ is the vector of independent control variables. The state variables vector $x$ can be expressed as follows:

$x=\left[P_{G S}, V_{L 1} \ldots V_{N L}, Q_{G 1} \ldots Q_{G N G}\right]$

$P_{G s}$ is the active power at slack generator; $V_{L}$ is the voltage magnitude of the load bus; $Q_{G}$ is the reactive power output for all generation units; $N L$ is number of load buses, $N G$ is the number of generation units. The control variables vector $\mu$ can be expressed as follows:

$\mu=\left[\begin{array}{c}P_{G 2} \ldots P_{G N G}, V_{G 1} \ldots V_{G N G} \\ Q_{C 1} \ldots Q_{C N C}, T_{1} \ldots T_{N T}\end{array}\right]$

$P_{G}$ is active power of the generator at PV buses; $V_{G}$ is the voltage magnitude for all the generation units; $Q_{C}$ is the shunt VAR compensation; $T$ is the tap settings of the transformer; $N C$ is the number of VAR compensators; $N T$ is the number of transformers. The equality constraints are the load flow equations:

$P_{G i}-P_{L i}=$

$V_{i} \sum_{j=1}^{N B} V_{j}\left(G_{i j} \cos \theta_{i j}+G_{i j} \sin \theta_{i j}\right)$

$Q_{G i}-Q_{L i}=$

$V_{i} \sum_{j=1}^{N B} V_{j}\left(G_{i j} \sin \theta_{i j}+G_{i j} \cos \theta_{i j}\right)$

$P_{G i}$ is the generated active power at bus $i ; Q_{G i}$ is the generated reactive power at bus $i ; P_{L i}$ is the active load demand at bus $i ; Q_{L i}$ is the reactive load demand at bus $i ; G_{i j}$ is the conductance between bus $i$ and $j ; B_{i j}$ is the susceptance between bus $i$ and $j$; $N B$ is the total number of buses. The inequality constraints include two parts

a- The inequality constraints of the control variables which consists of

- Upper and lower active power limit at PV bus $i$ except the slack bus

$P_{G i}^{\min } \leq P_{G i} \leq P_{G i}^{\max } \quad i=2, \ldots, N G$

- Upper and lower voltage magnitude limit of generator $i$ 
$V_{G i}^{\min } \leq V_{G i} \leq V_{G i}^{\max } \quad i=1,2,3, \ldots, N G$

- Upper and lower limit of shunt VAR compensator

$Q_{C i}^{\min } \leq Q_{C i} \leq Q_{C i}^{\max } \quad i=1,2,3, \ldots, N C$

- Upper and lower tap settings limit of transformer $i$

$T_{i}^{\min } \leq T_{i} \leq T_{i}^{\max } \quad i=1,2,3, \ldots, N T$

b- The inequality constraints of the control variables which consists of

- Upper and lower reactive power limit of generator $i$

$Q_{G i}^{\min } \leq Q_{G i} \leq Q_{G i}^{\max } \quad i=1,2, \ldots, N G$

- Upper and lower voltage limit at load bus $i$

$V_{L i}^{\min } \leq V_{L i} \leq V_{L i}^{\max } \quad i=1,2, \ldots, N L$

- Upper and lower active power of the slack generator $s$

$$
P_{G S}^{\min } \leq P_{G s} \leq P_{G S}^{\max }
$$

\section{Objective Functions}

Different Objective Functions of Optimal Power Flow have been used in this article to illustrate the efficiency of the proposed technique of Ant Colony Optimization. The various cases of objective functions are given as follows:

\subsection{Case 1: Generation fuel cost}

The total system fuel cost is modelled as the sum of the cost function of each generator. The generator fuel cost curves are modelled with smooth quadratic functions and measured by the unit $\$ / \mathrm{h}$ as shown in the equation (15).
Cost $=\sum_{i=1}^{N G} a_{i}+b_{i} P_{G i}+c_{i} P_{G i}^{2}$

where $N G$ is the number of thermal units, $P_{G i}$ is the active power generation at unit $i$ and $a_{i}, b_{i}$, $c_{i}$ are the cost coefficients of the $i$ th generator.

\subsection{Case 2: Voltage profile improvement}

The second objective function is to minimize the voltage deviation at the load buses from a specified voltage, which can be expressed as follows:

$V D=\sum_{i=1}^{N P Q}\left|V_{i}-1\right|$

Where $V D$ is the total voltage deviation at the load buses; $N P Q$ is the number of load buses and $V_{i}$ is the magnitude voltage at bus $i$.

\subsection{Case 3: Active power transmission losses}

In this case the required objective function is to minimize the active power transmission losses which is formulated as follows:

$$
P_{\text {loss }}=\sum_{k=1}^{N T L} G_{i j}\left(V_{i}^{2}+V_{j}^{2}-2 V_{i} V_{j} \cos \theta_{i j}\right)
$$

where, $G_{i j}$ the mutual conductance between bus $i$ and bus $j$ respectively; $V_{i}$ and $V_{j}$ representing the voltage magnitude at bus $i$ and bus $j$ respectively; $N T L$ is the number of transmission lines and $\theta_{i j}$ is voltage angle difference between buses voltages $i$ and $j$.

\section{Mechanism of Ant Colony Optimization}

Ant Colony Optimization imitates the behavior of real ant. Real ants can find the shortest possible 
route (path) between a food source and their nest by depositing the pheromone material. The pheromone material is a chemical substance produced by the ants evaporates with the time. It acts as a way of communication between the ants. This mechanism allowing the ants to choose the shortest possible route to search the food for the next movement. The pathway that has the highest pheromone concentration will be end up the target route $[6,7]$.

\section{State Transition Rule}

The ant choose the next node from the states of the second stage according to the state transition rule $P(r, s)$ as shown in equation (18)

$P_{(r, s)}^{k}=\left\{\begin{array}{c}\frac{\tau_{(r, s)}^{\alpha_{1}} \times \gamma_{(r, s)}^{\alpha_{2}}}{\sum \tau_{(r, s)}^{\alpha_{1}} \times \gamma_{(r, s)}^{\alpha_{1}}} \\ 0 \quad, \text { otherwise }\end{array} \quad r, s \in T\right.$

$\tau_{(r, s)}$ is the pheromone deposited (intensity of pheromone) between nodes $r$ and $s ; r$ is the previously node; $s$ is the currently node; $\gamma_{(r, s)}$ is the heuristic information which is the inverse of the distance between node $r$ and $s ; \alpha_{1}, \alpha_{2}$ are constants to control the influence of $\tau_{(r, s)}, \gamma_{(r, s)}$ respectively; $k$ is the ant number; $T$ is the set of paths that ant $k$ has not visited. The probability of selection a particular path $P_{(r, s)}^{k}$ increases with the value of the related pheromone trail $\tau_{(r, s)}$ and of the heuristic information value $\gamma_{(r, s)}$.

\section{Local updating rule}

When constructing a solution (implemented path), the ants will utilize a local pheromone update rule. This local pheromone is applied instantly after crossing the edge $(r, s)$ during the making of the path of control variables shown in equation (5). In others words, this update is satisfied after finishing the vector of control variables for a specified objective function.

$\tau_{(r, s)}=\rho \tau_{(r, s)}+\tau_{(r, s)}^{k}$

$\Delta \tau_{(r, s)}^{k}=1 /(\mathrm{CP} \times \mathrm{OF})$

where $(0<\rho \leq 1)$ is the persistence of the pheromone trail; $\Delta \tau_{(r, s)}^{k}$ is the amount of pheromone ant $k$ deposits on the path it has visited; $\mathrm{CP}$ is a larger positive constant and $\mathrm{OF}$ is the objective function [8].

\section{Global update rule}

Each tours (path) of ant represents a vector of control variables which has a specific objective function. After all ants complete their tours (path), the solution of population for the objective function is determined. The pheromone trails $(r, s)$ of the best ant tour that has minimum objective function is updated according to the global updating rule in equation (18)

$\tau_{(r, s)}=(1-\rho) \tau_{(r, s)}+\rho \Delta \tau_{(r, s)}^{b e s t}$

$\Delta \tau_{(r, s)}^{b s}=1 /(O F)^{b e s t}$

where $(O F)^{\text {best }}$ is best objective function based on best tour; The equations $(21,22)$ show the pheromone trail update for each $(r, s) \in L^{b s e t}$, where $L^{\text {best }}$ is best tour found since the start of algorithm. Both of evaporation and new deposit of pheromone are applies to $L^{\text {best }}$ edges [9].

\section{Ant Colony Optimization ACO in Constrained Optimal Power Flow}

In this application, the power system constraints are achieved by integration the control variables settings between the maximum and minimum values keeping the state variables in their limits. 
Figure (1) describes the ACO technique from the nest to the destination to find the optimal path for a specific objective function. The control variables are represented by the stages $(i=1, \ldots, n)$ while the candidate values between the minimum and maximum limits of each stage are represented by a set of the state nodes $(j=1, \ldots, D)$. The artificial ants will start to move from the nest (home colony) and it will pass through each stage (control variables), then stop at the destination (objective function). The ant chooses the state of each stage to complete one path. As example, the ant chooses the vector of control variables $\left[\mathrm{X}_{12}, \mathrm{X}_{23}, \mathrm{X}_{33}, \mathrm{X}_{41}, \mathrm{X}_{52}, \ldots, \mathrm{X}_{\mathrm{n} 2}\right]$ to complete one path for specific objective function.

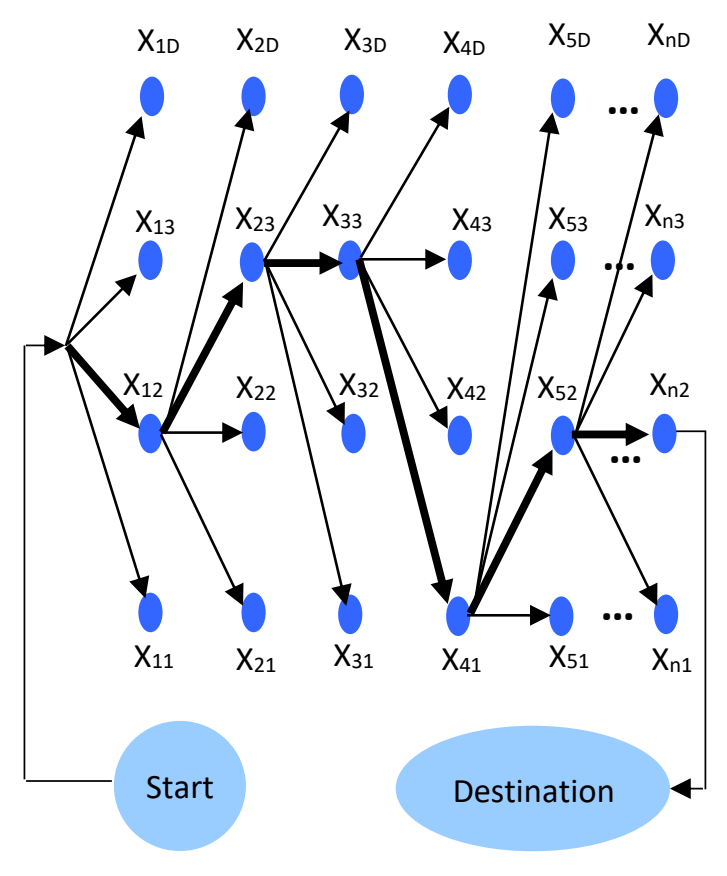

Figure 1. ACO for the OPF problem

\section{The steps of the ACO algorithm}

\section{Step 1: Initialization}

Initialize the population of the Ant Colony Optimization where the number of population is equal to the number of ants. Each vector of population is represent by a path of the ant, where each path of ant is equal to vector of control variable. The population is equal to a set of control variables.

\section{Step 2: Create the first node}

The first node is chosen randomly from the states for the first stage (first stage represents first control variable)

\section{Step 3: Apply state transition rule}

The state transition rule $P_{(r, s)}$ in equation (18) is applied for each state to select the next node, it is applied through considering the previously selected state (r) in step 2 . The path to be chosen is determined based on the following points:

a- The roulette-wheel selection procedure is applied to choose a particular path for each ant (k) as shown in the step (b, c, d) below:

b- Generate a random number in the range $(0,1)$. This number is different for each ant.

c- Find the ranges of cumulative probability connected with different paths based on equation 15 .

d- Compare the cumulative probability range in point (c) with the random number generated in point $b$, if it is larger than the random number, this means that the respective state (s) can be accepted as a path for the ant. Otherwise, it will be ignored.

This selection will be repeat for all paths (s) from the current control variable state.

\section{Step 4: Solution for each ant}

After the paths have been determined for all the ants. The total objective function traversed by each ant must be calculated, this total objective function is used to update the pheromone.

\section{Step 5: The pheromone update rule}

Update the local and global pheromone level using the equations $(19,20)$ and $(21,22)$ respectively.

\section{Step 6: Termination condition}

The ACO algorithm stops the iteration after the maximum number of iterations are finished and the tour is completed by all the ants. After the termination condition has been satisfied and all 
the ants taken the tour, the best solutions provided by the ants are presented.

\section{The application and result}

The IEEE 30 bus system in figure 2 has been used to test different single objective optimal power flow problem based on the Ant Colony Optimization technique. This system has 6 generators at buses 1, 2, 5, 8, 11 and 13 with four transformers at lines 4-12, 6-9, 6-10 and 28-27, also this system has nine shunt VAR compensation at buses 10, 12, 15, 17, 20, 21, 23, 24 and 29. Therefore, this system has 24 control variables. Table 1 represent the cost coefficients of the generation units.

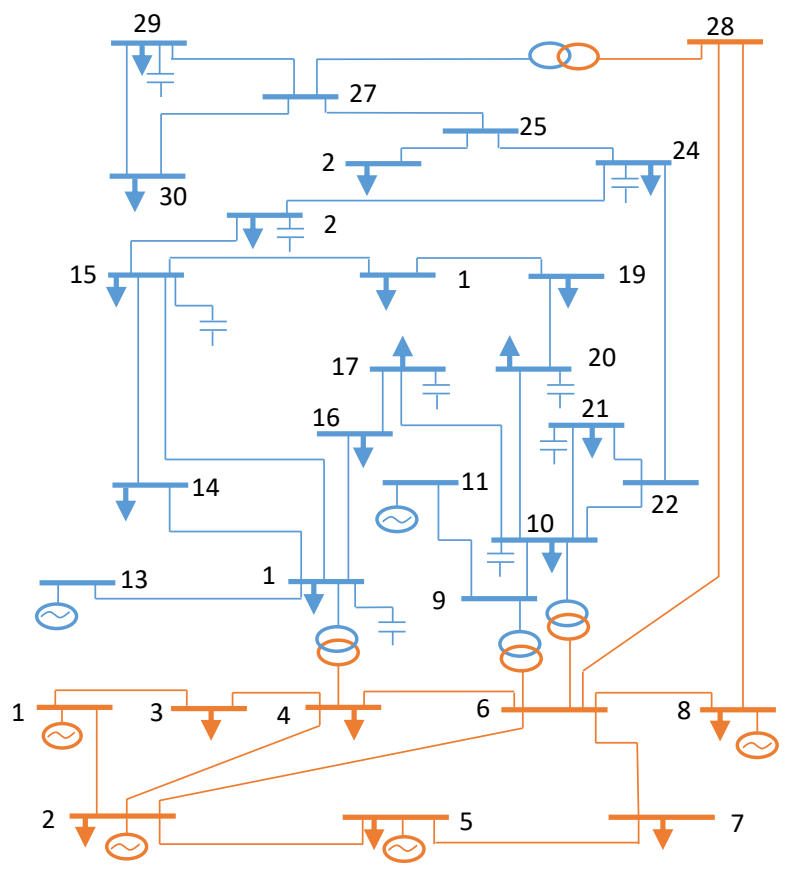

Figure 2. IEEE-30 Bus System

Table 1. Generator cost coefficients

\begin{tabular}{cccc}
\hline Unit & $a_{i}$ & $b_{i}$ & $c_{i .}$ \\
number $(i)$ & $(\$ / \mathrm{h})$ & $(\$ / M W h)$ & $\left(\$ / M W^{2} \mathrm{~h}\right)$ \\
1 & 0 & 2.00 & 0.00375 \\
2 & 0 & 1.75 & 0.0175 \\
5 & 0 & 1.00 & 0.0625 \\
8 & 0 & 3.25 & 0.0083 \\
11 & 0 & 3.00 & 0.0250 \\
13 & 0 & 3.00 & 0.0250 \\
\hline
\end{tabular}

Table 2 shows the optimal setting of the Control Variables (C.V) with its minimum objective functions (case1: fuel cost $(\$ / \mathrm{h})$, case2: voltage deviation (pu) and case3: active power losses (MW) using the propose algorithm of the Ant Colony Optimization ACO technique.

Table 2. Control Variables( C.V) for the three cases of objective function

\begin{tabular}{|c|c|c|c|c|c|c|}
\hline C.V & Mn. & Mx. & Initial & Case1 & Case2 & Case3 \\
\hline$P_{G 2}$ & 20 & 80 & 80 & 48.9 & 61.44 & 68.09 \\
\hline$P_{G 5}$ & 15 & 50 & 50 & 22.68 & 26.71 & 48.83 \\
\hline$P_{G 8}$ & 10 & 35 & 20 & 19.59 & 15.47 & 34.87 \\
\hline$P_{G 11}$ & 10 & 30 & 20 & 10.7 & 26.96 & 26.35 \\
\hline$P_{G 13}$ & 12 & 40 & 20 & 13.17 & 36.37 & 38.92 \\
\hline$V_{G 1}$ & 0.95 & 1.1 & 1.05 & 1.085 & 1.015 & 1.086 \\
\hline$V_{G 2}$ & 0.95 & 1.1 & 1.04 & 1.064 & 1.006 & 1.078 \\
\hline$V_{G 5}$ & 0.95 & 1.1 & 1.01 & 1.047 & 1.016 & 1.068 \\
\hline$V_{G 8}$ & 0.95 & 1.1 & 1.01 & 1.035 & 1.009 & 1.062 \\
\hline$V_{G 11}$ & 0.95 & 1.1 & 1.05 & 1.065 & 1.083 & 1.097 \\
\hline$V_{G 13}$ & 0.95 & 1.1 & 1.05 & 1.056 & 0.995 & 1.091 \\
\hline$T_{4-12}$ & 0.9 & 1.1 & 1.078 & 1.058 & 0.945 & 1.016 \\
\hline$T_{6-9}$ & 0.9 & 1.1 & 1.069 & 1.091 & 1.079 & 1.048 \\
\hline$T_{6-10}$ & 0.9 & 1.1 & 1.032 & 0.962 & 0.925 & 0.973 \\
\hline$T_{28-2}$ & 0.9 & 1.1 & 1.068 & 1.043 & 0.947 & 0.981 \\
\hline$Q_{C 10}$ & 0 & 5 & 0 & 2.044 & 2.553 & 4.674 \\
\hline$Q_{C 12}$ & 0 & 5 & 0 & 2.827 & 0.619 & 0.244 \\
\hline$Q_{C 15}$ & 0 & 5 & 0 & 4.798 & 3.673 & 2.376 \\
\hline$Q_{C 15}$ & 0 & 5 & 0 & 4.595 & 4.398 & 4.183 \\
\hline$Q_{C 17}$ & 0 & 5 & 0 & 4.940 & 4.814 & 4.296 \\
\hline$Q_{C 20}$ & 0 & 5 & 0 & 1.416 & 2.488 & 2.318 \\
\hline$Q_{C 21}$ & 0 & 5 & 0 & 0.038 & 4.774 & 3.837 \\
\hline$Q_{C 23}$ & 0 & 5 & 0 & 4.308 & 3.075 & 2.541 \\
\hline$Q_{C 29}$ & 0 & 5 & 0 & 4.359 & 58 & 1.440 \\
\hline \multicolumn{7}{|c|}{ Objective function } \\
\hline \multicolumn{3}{|c|}{$\begin{array}{l}\text { Case } 1: \text { Fuel Cost } \\
(\$ / h)\end{array}$} & 902.0 & 801.4 & 843.6 & 930.4 \\
\hline \multicolumn{3}{|c|}{$\begin{array}{l}\text { Case 2: Voltage } \\
\text { deviation }(\mathrm{pu})\end{array}$} & 1.154 & 0.429 & 0.119 & 1.187 \\
\hline \multicolumn{3}{|c|}{$\begin{array}{l}\text { Case 3: Power } \\
\text { losses (MW) }\end{array}$} & 5.839 & 9.221 & 7.376 & 3.417 \\
\hline
\end{tabular}

According to Table 2, the total fuel cost is substantially decreased from initial value of 902.0 $\$ / \mathrm{h}$ to optimal value of $801.4 \$ / \mathrm{h}$ with reduction of $(11.15 \%)$. The voltage deviation decreasing from initial value of $1.1547 \mathrm{pu}$ to optimal value of $0.119 \mathrm{pu}$ with a reduction of $89.69 \%$. The active 
power transmission line losses is significantly decreased by $(41.48 \%)$ from initial value of 5.839 MW to optimal value of 3.417 MW. The variation of the three objective functions (fuel cost, voltage deviation and active lower losses) with number of iteration are illustrated in the Figures 3, 4 and 5 respectively.

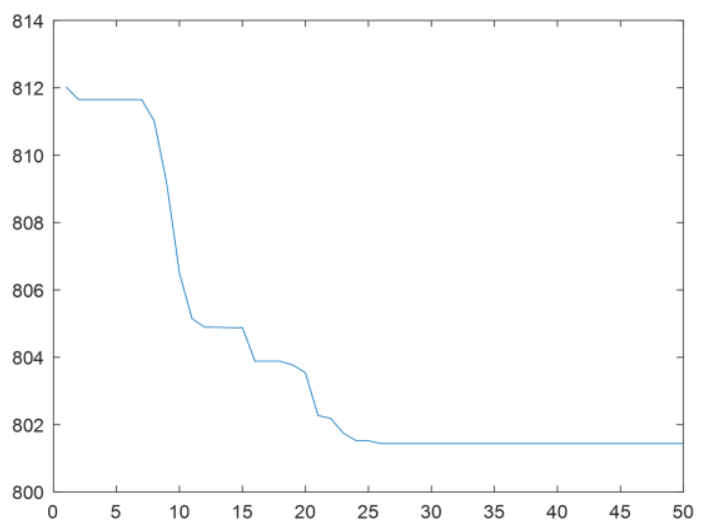

Figure 3. Fuel Cost $(\$ / h)$ with iteration number

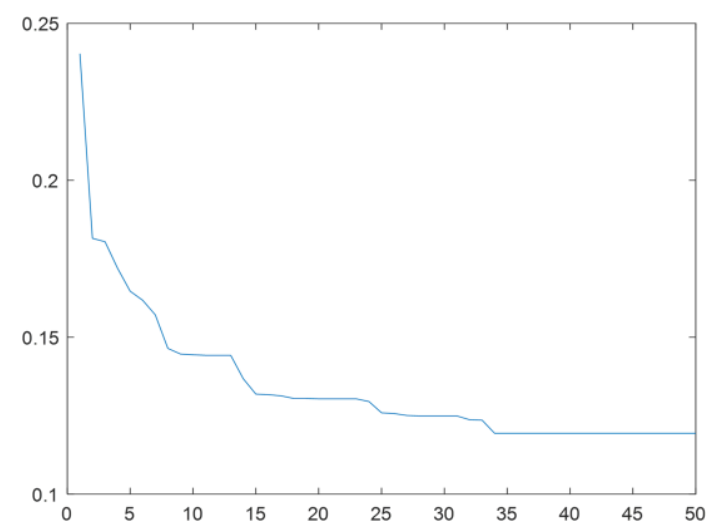

Figure 4. Voltage deviation (pu) with iteration number

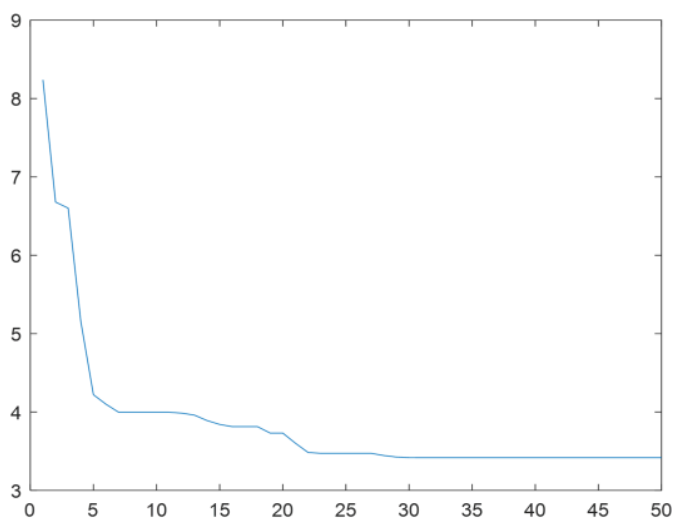

Figure 5. Active losses (MW) with iteration number
Table 3 shows the state variables of the generators reactive power and the active power of the slack generator which they not exceed their limits after applying the ACO technique to solve the OPF problem. Therefore, the condition of the constraint OPF are satisfied.

Table 3. Generators reactive power constraint and slack active power constraint

\begin{tabular}{cccccc}
\hline Units & Min & Max & Case1 & Case2 & Case3 \\
1 & -20 & 200 & 10.5 & -8.0 & 3.54 \\
3 & -20 & 100 & 12.51 & -1.98 & 9.74 \\
5 & -15 & 80 & 42.92 & 60.15 & 36.38 \\
8 & -15 & 60 & 17.07 & 54.89 & 24.52 \\
11 & -10 & 50 & 29.79 & 42.39 & 24.44 \\
13 & -15 & 60 & 25.99 & -10.15 & 20.17 \\
slack & 50 & 200 & 177.4 & 123.81 & 69.760 \\
active & & & & & \\
power & & & & & \\
\hline
\end{tabular}

Also the constraint of the state variable of the load voltage after applying ACO technique to solve the OPF problem are within the minimum and maximum limits (0.9 and 1.1 respectively) as shown in the Figure 7.

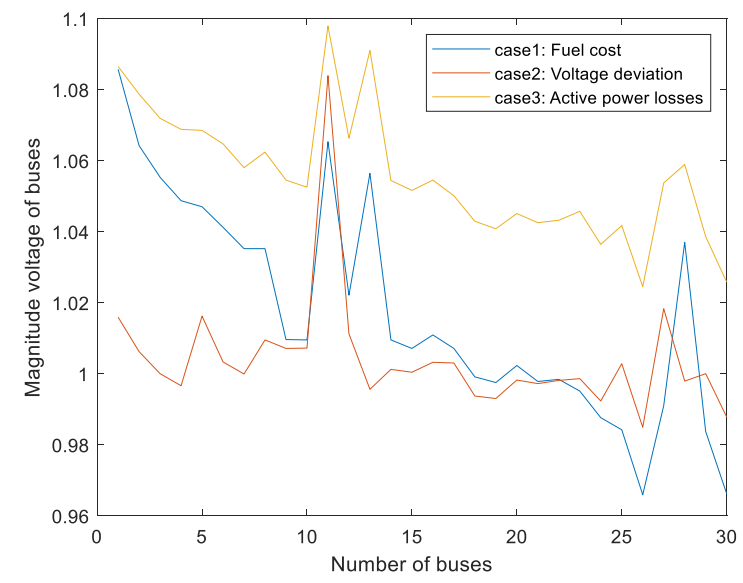

Figure 7. Magnitude voltage in pu of IEEE 30 bus

The propose algorithm gives good result when compare with other optimization techniques used the same data and test system of IEEE 30 bus as shown in Tables (4, 5 and 6). 
Table 4. Comparison of voltage deviation VD with other optimization techniques

$\begin{array}{ccc}\text { Ref. } & \text { Optimization techniques } & \begin{array}{c}\text { VD } \\ (\mathrm{pu})\end{array} \\ {[19]} & \text { Differential Evolution } & 0.19 \\ {[20]} & \text { Particle Swarm Optimization } & 0.1334 \\ {[20]} & \text { Moth-flame Algorithm } & 0.0976 \\ {[21]} & \text { Black-hole-Based } & 0.1262 \\ \text { propose } & \text { Optimization } \\ \text { method } & \text { Ant Colony Optimization } & \mathbf{0 . 1 1 9 4} \\ \end{array}$

Table 5. Comparison of active power losses $P_{\text {loss }}$ with other optimization technique

\begin{tabular}{ccc}
\multicolumn{3}{c}{ with other optimization technique } \\
\hline Ref. & Optimization techniques & $\begin{array}{l}P_{\text {loss }} \\
\text { (MW) }\end{array}$ \\
{$[22]$} & conventional interior point & 5.101 \\
{$[19]$} & Particle Swarm Optimization & 5.092 \\
{$[23]$} & Differential Evolution & 4.760 \\
{$[24]$} & Differential Evolution & 4.720 \\
{$[14]$} & Flower Pollination Algorithm & 3.5661 \\
{$[21]$} & black-hole-Based & 3.503 \\
propose & Ant Colony Optimization \\
method & & $\mathbf{3 . 4 1 7 1}$ \\
\hline
\end{tabular}

Table 6. Comparison of fuel cost with other optimization techniques

\begin{tabular}{ccc}
\hline Ref. & Optimization techniques & $\begin{array}{c}\text { Fuel cost } \\
(\$ / \mathrm{h})\end{array}$ \\
{$[11]$} & Gradient & 804.85 \\
{$[12]$} & Ant Colony Optimization & 803.12 \\
{$[13]$} & Differential Evolution & 803.05 \\
{$[13]$} & Imperialistic Competitive & 802.88 \\
{$[14]$} & Flower Pollination Algorithm & 802.79 \\
{$[15]$} & Improved PSO & 802.63 \\
{$[15]$} & Evolutionary Programming & 802.62 \\
{$[16]$} & modified differential evolution & 802.37 \\
{$[17]$} & modified shuffle frog leaping & 802.28 \\
{$[18]$} & Particle Swarm Optimization & 801.66 \\
propose & Ant Colony Optimization & $\mathbf{8 0 1 . 4 3}$ \\
method & & \\
\hline
\end{tabular}

This minimization of the objective function based on ACO technique indicates the effectiveness of the proposed technique to solve OPF problem

\section{Conclusion}

This article used the Ant Colony Optimization ACO to solve the Optimal Power Flow OPF problems. One test system and three cases of objective functions were tested to determine the efficiency of the suggested algorithm. The proposed algorithm chooses the optimal control variables to minimize these different Objective functions (fuel cost, voltage deviation and active power losses), while satisfying different types of constraints. On the IEEE 30-bus test system, the practicability of the expected approach has been checked. The acquired outcomes from this technique were good when compared with other optimization techniques that used to solve the OPF problem in the literature of this article. It proved to be effective, accurate, successful, acceptable solutions and has low implementation time in solving optimal power flow problem. The proposed algorithm ACO was executed step by step using the programming of $M$ file in the MATLAB software.

\section{Acknowledgements}

The authors would like to acknowledge the department of Electrical Engineering, Collage of Engineering, Mustansiriyah University for their encourage and support.

\section{Conflict of interest}

The authors identify and declare that the publication of this article cause no conflict of interest.

\section{References}

1. V. Raviprabakaran, R. Subramania, (2018). "Enhanced ant colony optimization to solve the optimal power flow with ecological emission". International Journal of Systems Assurance Engineering and Management, Vol. 9, pp. 58-65.

2. J. Carpienter, (1962) "Contribution e l'étude do Dispatching Economique". Bulletin Society Française Electriciens, Vol. 3, pp. 96104. 
3. B. Gasbaoui, B. Allaoua, (2009). "Ant Colony Optimization Applied on Combinatorial Problem for Optimal Power Flow Solution". Leonardo Journal of Sciences, vol. 14, pp. 117.

4. J. A. Momoh, M. E. El-Hawary and R. Adapa, (1999). "A review of selected optimal power flow literature to 1993 part I: NonLinear and quadratic programming approaches". IEEE Transactions on Power Systems, Vol. 14, pp. 96-104.

5. J. A. Momoh, M. E. El-Hawary and R. Adapa, (1999). "A review of selected optimal power flow literature to 1993 part II: Newton, linear programming and interior point methods". IEEE Transactions on Power Systems, Vol. 14, pp. 105-111.

6. M. Dorigo, V. Maniezzo, and A. Colorni, (1996). "Ant system: optimization by a colony of cooperating agents". IEEE Trans. Syst., Man, Cybern. B, Vol. 26, No. 1, pp. 29-41.

7. M. Dorigo and L. M. Gambardella, (1997). "Ant colony system: A cooperative learning approach to the travelling salesman problem". IEEE Trans. Evolutionary Computation, Vol. 1, pp. 53-66.

8. John G. Vlachogiannis, Nikos D. Hatziargyriou, and Kwang Y. Lee, (2005). "Ant Colony System-Based Algorithm for Constrained Load Flow Problem". IEEE Transactions on Power systems, Vol. 20, pp. 1241-1249.

9. L. Wang, J. Shen and G. Beydoun, (2013) " Enhanced ant colony algorithm for cost-aware data-intensive service provision". IEEE Ninth World Congress on Services, pp. 227-234.

10. R. Cabadag, B. Turkay, (2013). "Heuristic methods to solve optimal power flow problem," Istanbul University-Journal of Electrical \& Electronics Engineering". Vol. 13, pp. 1653-1659.

11. K. Y. Lee, Y. M. Park, J. L. Qrtiz, (1985) "A united approach real and reactive power dispatch". IEEE Transactions on Power Apparatus and Systems, Vol. PAS-104, pp.1147-1153.

12. B. Allaoua and A. Laoufi, (2009). "Optimal power flow solution using ant manners for electrical network". Advances in Electrical and Computer Engineering, Vol. 9, No. 1, pp. 34-40.

13. G. N. Reddy, S. S. Dash, S. Sivanagaraju, Ch. V. Suresh, (2014). "Economic load dispatch using imperialistic competitive algorithm: An effect of control variables". International Journal of Engineering and Advanced Technology (IJEAT), Vol. 3, Issue 6.

14. Xin-She Yang, (2012). "Flower pollination algorithm for global optimization". International conference on unconventional computing and natural computation, Vol. 7445, Springer Lecture Notes in Computer Science, Berlin, Heidelberg, pp. 240-249.

15. B. Yang, Y. Chen, Z. Zhao, Q. Han, (2006). "Solving optimal power flow problems with improved particle swarm optimization". Proceedings of the 6th World Congress on Intelligent Control and Automation, Vol. 2, pp. 7457-7461.

16. S. Sayah, K. Zehar, (2008). "Modified differential evolution algorithm for optimal power flow with non-smooth cost function". Energy conversion and Management, Vol. 49, pp. 3036-3042.

17. T. Niknam, MR. Narimani, M. Jabbari, AR. Malekpour, (2008). "A modified shuffle frog leaping algorithm for multi-objective optimal power flow" Energy, Vol. 36, pp. 6420-6432.

18. L. Al-Bahrani, V. Dumbrava, (2016). "Optimal power flow based on particle swarm optimization". University POLITEHNICA OF Bucharest Scientific Bulletin Series CElectrical Engineering and Computer science, Vol. 78, pp. 253-264. 
19. R. K. Verma, H. Singh, L. Srivastava, (2012). "Optimal power flow using differential evolution algorithm with conventional weighted sum method". International Journal of Computational Engineering Research (IJCER), Vol. 2, No.3.

20. M. Ebeed, S. Kamel, H. Youssef, (2016). "Optimal Setting of STATCOM Based on Voltage Stability Improvement and Power Loss Minimization Using Moth-flame Algorithm". Power System Conference, MEPCON, International Middle-East, IEEE.

21. H. Bouchekara, (2014). "Optimal power flow using black-hole-based optimization approach". Applied Soft Computing, Vol. 24, pp. 879-888.

22. M. Varadarajan, K.S. Swarup, (2008). "Differential evolutionary algorithm for optimal reactive power dispatch". Elsevier, Electrical Power Systems Research, Vol. 30, pp. 435-441.

23. B. Mahanta, B. Goswami, (2015). "An efficient differential evolutionary approach to optimal reactive power dispatch with voltage profile improvement". International Research Journal of Engineering and Technology (IRJET), Vol. 2, No. 3.

24.P. A. Jeyanthy, D. Devaraj, (2010). "Optimal reactive power dispatch for voltage stability enhancement using real coded genetic algorithm". International Journal of Computer and Electrical Engineering, Vol. 2, No. 4. 